\title{
Short-Term Plasticity Shapes the Response to Simulated Normal and Parkinsonian Input Patterns in the Globus Pallidus
}

\author{
Jesse E. Hanson and Dieter Jaeger \\ Department of Biology, Emory University, Atlanta, Georgia 30322
}

Basal ganglia structures show strong activity modulation during movement and synchronous bursting in Parkinson's disease. Recent work has shown that short-term synaptic plasticity (STP) can play an important role in the effect of temporal activity patterns on postsynaptic targets. To determine the role of STP in the subthalamic nucleus (STN) to globus pallidus (GP) connection, which has been suggested to underlie rhythmical bursting in Parkinson's disease, we first measured STP using trains of electrical input stimulation in vitro. We found that STN inputs to GP typically show both facilitation and depression with input frequencies of $10-100 \mathrm{~Hz}$ and that facilitation is dominant for the first few inputs in a train but that depression takes over subsequently. We quantified the strength and time course of facilitation and depression using a computational

The current network model of basal ganglia circuitry explains pathological conditions, including Parkinson's disease, as resulting from an imbalance in the activity level between the direct and indirect [via subthalamic nucleus (STN)] pathways from the striatum to output structures (Albin et al., 1989; DeLong, 1990; Smith et al., 1998). Despite the proven conceptual and clinical usefulness of this model, it is limited by its simple linear representation of the control of neural activity by synaptic input rates. To achieve a more realistic and powerful understanding of how the basal ganglia function, or dysfunction, the influence of dynamically modulated synaptic activity on spike rates and patterns needs to be incorporated into this model (Wichmann and DeLong, 1996, 1998).

One important mechanism by which the influence of a temporal pattern of presynaptic activity on spike output can be modulated is provided by short-term plasticity (STP), i.e., a change in synaptic response properties based on inputs in the preceding few seconds (Abbott et al., 1997; Varela et al., 1997). STP has been found in neurons throughout the brain and can involve depressing influences, facilitating influences, or both simultaneously (Bonci and Malenka, 1999; Dittman et al., 2000; Hempel et al., 2000). The use of models in which STP is quantified by dynamic variables related to facilitation and depression has allowed accurate prediction of response amplitudes to input patterns at various frequencies (Varela et al., 1997; Dittman et al., 2000; Hempel et al., 2000).

The functional impact of STP in the basal ganglia is generally

\footnotetext{
Received Aug. 8, 2001; revised Feb. 20, 2002; accepted March 21, 2002.

This work was supported by National Institutes of Health Grants NS39852 and MH12999.

Correspondence should be addressed to Dieter Jaeger, Emory University, Department of Biology, 1510 Clifton Road, Atlanta, GA 30322. E-mail: djaeger@ emory.edu.

Copyright (C) 2002 Society for Neuroscience $\quad 0270-6474 / 02 / 225164-09 \$ 15.00 / 0$
}

model of STP. Using the STP model, we constructed synaptic conductance patterns of normal and Parkinsonian STN activity and applied these conductances to GP neurons in vitro using the technique of dynamic clamping. We show that STP controls the slope and shape of the function describing the steady-state level of GP neuron firing in response to different levels of STN input. In addition, we show that STP modulates responses of GP neurons to bursts and pauses in the input pattern. These findings indicate that STP plays an important role in modulating both spike rates and temporal patterns of GP activity in the normal state, as well as in Parkinson's disease.

Key words: subthalamic nucleus; synaptic depression; synaptic facilitation; temporal coding; basal ganglia; dynamic clamp unknown. Diverse patterns of temporal activity that could be significantly modulated by STP exist throughout the basal ganglia, however. These patterns include phasic rate changes related to the execution of sensorimotor tasks (Georgopoulos et al., 1983; DeLong et al., 1985; Gardiner and Kitai, 1992; Wichmann et al., 1994; Jaeger et al., 1995; Turner and Anderson, 1997), as well as shifts from asynchronous, irregular firing during wakefulness to synchronous, bursty firing during sleep (Urbain et al., 2000), anesthesia (Magill et al., 2000), and Parkinson's disease (Filion and Tremblay, 1991; Bergman et al., 1994, 1998; Nini et al., 1995). These patterns are believed to be particularly influenced by a feedback interaction between the globus pallidus (GP) and the subthalamic nucleus (Berns and Sejnowski, 1998; Plenz and Kitai, 1999; Magill et al., 2000). Furthermore, neurons in the STN can generate bursting activity intrinsically (Beurrier et al., 1999), and STN bursting is prominent in animal models of Parkinson's disease (Bergman et al., 1994). Therefore, as a first step toward understanding the functional impact of STP on temporal patterns in the basal ganglia, we examined how STP dynamically regulates the connection from the STN to the GP during patterns of activity characteristic of normal and parkinsonian states. First, we measured the STP of postsynaptic excitatory currents in the GP with temporal patterns of electrical stimulation during whole-cell recording in vitro. We subsequently determined parameters of a computational model describing STP with dynamic variables (Varela et al., 1997) to fit the STP in this pathway. Finally, we examined the effect of STP in this pathway on GP spiking patterns by applying simulated STN input patterns to GP neurons in vitro using the technique of dynamic current clamping (Robinson and Kawai, 1993; Sharp et al., 1993; Jaeger and Bower, 1999).

\section{MATERIALS AND METHODS}

Physiological recordings. Frontal slices through the GP $(300 \mu \mathrm{m})$ were prepared from 16- to 25 -d-old male Sprague Dawley rats $(n=45)$ 
(Charles River Laboratories, Wilmington, MA). All animal procedures complied fully with the National Institutes of Health guidelines on animal care and use. Whole-cell recordings were obtained with an Axoclamp-2B amplifier (Axon Instruments, Foster City, CA) from visually identified neurons at a recording temperature of $32^{\circ} \mathrm{C}$. The slice medium contained (in mM): $124 \mathrm{NaCl}, 3 \mathrm{KCl}, 1.2 \mathrm{KH}_{2} \mathrm{PO}_{4}, 26 \mathrm{NaHCO}_{3}$, $2 \mathrm{CaCl}_{2}, 1.9 \mathrm{MgSO}_{4}$, and 20 glucose. Electrodes were filled with (in mM): 140 K-gluconate, 10 HEPES, $10 \mathrm{NaCl}, 0.2$ EGTA, 4 MgATP, 0.4 NaGTP, 0.05 spermine, and 5 glutathione. In some control experiments, K-gluconate was replaced with Cs-methanesulfonate, and $10 \mathrm{~mm} \mathrm{QX-314}$ (Sigma. St. Louis, MO) was added. Electrode resistance ranged between 6 and $12 \mathrm{M} \Omega$. Electrodes were coated with Sylgard (model 184; Dow Corning, Corning, NY).

Electrical stimulation. Inhibitory synaptic transmission was blocked using $40 \mu \mathrm{M}$ picrotoxin. Glass pipette electrodes identical to the recording electrodes filled with $0.5 \mathrm{M} \mathrm{NaCl}$ were placed in the slice within 250 $\mu \mathrm{m}$ of the cell body. Bipolar stimuli of $0.2 \mathrm{msec}$ duration and $20-80 \mathrm{~V}$ amplitude were delivered using stimulus-isolation units (World Precision Instruments, Sarasota, FL). These electrodes provided very focal stimulation of only a few fibers, as evidenced by a change or disappearance of responses when moving the stimulation electrode by $5-10 \mu \mathrm{m}$. The elicited fast EPSCs were deemed to represent, in the vast majority, STN input, which is by far the dominant source of excitation on GP neurons (Shink and Smith, 1995; Shink et al., 1996; Smith et al., 1998). Because some fibers have been found to project to the GP from the thalamus (Deschenes et al., 1996) and cortex (Naito and Kita, 1994), EPSCs elicited at some stimulation sites may be derived from these pathways. Different kinds of EPSCs based on time course or amplitude could not be distinguished, however. To create stimulation patterns that resembled in vivo STN neuron spike trains, irregular stimulus trains with different mean frequencies were constructed using a gamma distribution with an absolute refractory period of $3 \mathrm{msec}$. One full stimulus set included $10-15$ repetitions of $10 \mathrm{sec}$ of mean $10 \mathrm{~Hz}$ activity, $5 \mathrm{sec}$ of $20 \mathrm{~Hz}$ activity in five bursts, $2 \mathrm{sec}$ of $50 \mathrm{~Hz}$ in four bursts, and $1 \mathrm{sec}$ of $100 \mathrm{~Hz}$ stimulation in two bursts. Activity bursts lasted $\sim 500 \mathrm{msec}$ and were separated by $500 \mathrm{msec}$ pauses. Pauses of at least $10 \mathrm{sec}$ separated the presentation of different stimulation frequencies.

Quantitative plasticity model. To quantify STP in the STN-GP connection, we modified a simple mathematical model that can fit physiological STP data without reference to the actual mechanisms underlying STP (Varela et al., 1997). This model calculates response amplitude, $A$, as the product of a facilitation variable, $F$, and a depression variable, $D$ $(A=F \times D$, where $F>1$ and $D<1)$. In our version of this model, the facilitation variable, $F$, is increased by multiplication with an increment factor, Inc $\mathrm{F}_{\mathrm{F}}$, at each time of synaptic activation $\left(F^{\prime}=F \times \operatorname{Inc}_{\mathrm{F}}\right.$, where Inc $_{\mathrm{F}}>1$ ) and decays exponentially with a time constant, $\tau_{\mathrm{F}}$, afterward. In addition, the growth of the $F$ variable was limited by an upper bound. To implement the upper bound on $F, F$ is incremented each time a stimulus occurs, as follows: $F^{\prime}=F \times$ Inc $_{\mathrm{FB}}$, where $\operatorname{Inc}_{\mathrm{FB}}=1+\left(\mathrm{Inc}_{\mathrm{F}}-\right.$ $1) \times\left(F_{\text {bound }}-F\right) /\left(F_{\text {bound }}-1\right)$. This implementation smoothly reduces Inc $_{\mathrm{FB}}$ from Inc $_{\mathrm{F}}$ to 1 as the upper bound for $F$ is reached. Tests were performed in which $F_{\text {bound }}$ was varied, and we found that a value of 5 allowed the best fits of the model to the data across the entire data set. The depression variable, $D$, is decreased by multiplication with a factor, Inc $_{\mathrm{D}}$, at each time of synaptic activation $\left(D^{\prime}=D \times\right.$ Inc $_{\mathrm{D}}$, where $\operatorname{Inc}_{\mathrm{D}}<$ 1 ) and decays with a time constant of $\tau_{\mathrm{D}}$ (see Fig. 2). The optimal values of Inc $\mathrm{F}_{\mathrm{F}}, \tau_{\mathrm{F}}, \mathrm{Inc}_{\mathrm{D}}$, and $\tau_{\mathrm{D}}$ to fit the data for each neuron were determined with automated optimization routines implemented in MatLab (MathWorks, Natick, MA). The root mean square error between model predictions and observed amplitudes across the entire data set for each neuron was minimized as follows. The region of the global minimum in the four-dimensional parameter space $\left(\operatorname{Inc}_{\mathrm{F}}, \operatorname{Inc}_{\mathrm{D}}, \tau_{\mathrm{F}}\right.$, and $\left.\tau_{\mathrm{D}}\right)$ was found using a genetic algorithm (Houck et al., 1995), and the local minimum within this identified region of parameter space was found using a simplex search (MatLab optimization toolbox). To confirm that this protocol arrived at the optimal set of parameters for each neuron, each data set was fit to the model with at least 10 different random seeds, such that the genetic algorithm evolved solutions based on different initial populations. For each neuron, this protocol resulted in the same optimal fit of the model to the experimental data with each random seed.

Dynamic current clamping. The modulation that STP may cause on the spike output of a GP neuron in vivo is dependent on the temporal sequence of inputs received. Such inputs include an asynchronous baseline, as well as specific signal patterns. The ensuing high-conductance state of baseline synaptic inputs leads to specific synaptic integration properties that cannot be extrapolated from the effect of single inputs (Jaeger et al., 1997; Jaeger and Bower, 1999; Destexhe et al., 2001). Thus, to examine how synaptic inputs control spike output, one would like to measure simultaneously all synaptic inputs, as well as spike output. This is not possible with natural synaptic input, but the same conductance patterns can be generated artificially with the technique of dynamic clamping. This technique consists of a fast-feedback cycle, in which the simulated synaptic current $\left(I_{\mathrm{s}}\right)$ is updated from the in vivo-like whole-cell excitatory and inhibitory synaptic conductances $\left(G_{\text {ex }}\right.$ and $\left.G_{\text {in }}\right)$ according to the equation $I_{\mathrm{s}}=G_{\mathrm{ex}}\left(V_{\mathrm{m}}-E_{\mathrm{ex}}\right)+G_{\mathrm{in}}\left(V_{\mathrm{m}}-E_{\mathrm{in}}\right)$, where $E_{\mathrm{ex}}$ and $E_{\mathrm{in}}$ are the reversal potentials of excitation and inhibition, respectively. Therefore, this current is time varying, depending on the present level of depolarization in the cell. In the present study, dynamic clamping was implemented using custom-made software running under the DOS operating system on a personal computer. The fast-feedback component of the program was written in assembly and was tested to perform at rates up to $20 \mathrm{kHz}$. Dynamic clamping was used to inject a current $\left(I_{\mathrm{s}}\right)$ that mimics the synaptic current of 100 excitatory inputs in the presence of an unvarying baseline of inhibitory conductance. As found in other cell types (Jaeger and Bower, 1999; Gauck and Jaeger, 2000), excitatory input without a balance of inhibition could not lead to realistic GP firing patterns, because these neurons have intrinsic pacemaker activity (Kita and Kitai, 1991) and fire too fast with pure excitation. The inhibitory conductance was held at a constant value during all patterns of excitatory conductance to isolate the effect of excitatory input patterns on output spiking. Endogenous excitatory and inhibitory synapses in the slice were blocked with $200 \mu \mathrm{M}$ AP-5, $10 \mu \mathrm{M}$ CNQX, and $40 \mu \mathrm{M}$ picrotoxin. The reversal potentials of the excitatory $\left(E_{\mathrm{ex}}\right)$ and inhibitory $\left(E_{\mathrm{in}}\right)$ synaptic currents were set to 0 and $-70 \mathrm{mV}$, respectively, on the basis of a linear regression analysis of measurements of IPSCs and EPSCs in GP at different holding potentials (data not shown). The recorded membrane potential $\left(V_{\mathrm{m}}\right)$ and the injected current were updated with a frequency of $10 \mathrm{kHz}$. The excitatory and inhibitory conductances $\left(G_{\mathrm{ex}}\right.$ and $\left.G_{\text {in }}\right)$ were calculated before the experiments. A single excitatory input element was simulated as a dual-exponential function with a $\tau_{1}$ of $5 \mathrm{msec}$ and a $\tau_{2}$ of 12 msec: $g_{\text {ex }}=g_{\max } /\left(\tau_{2} / \tau_{1}\right) \times\left[e^{-\left(\mathrm{t} / \tau_{2}\right)}-e^{-\left(\mathrm{t} / \tau_{1}\right)}\right]$. These time constants were selected to match the time course of experimentally observed spontaneous EPSCs recorded in the presence of picrotoxin. The unitary amplitude of excitatory conductances was adjusted between 0.5 and 1.5 $\mathrm{nS}$ to match the magnitude of injected current to qualitative observations of the input conductance of each neuron. These values also approximate the magnitude of synaptic conductances measured during stimulation experiments. The ratio of $G_{\mathrm{ex}}$ to $G_{\text {in }}$ was adjusted within the range of $0.2-2$ to achieve a dynamic range of firing across the entire set of excitatory input conductances for each neuron without cessation of spiking attributable to hyperpolarization or depolarization block. The responses of GP neurons to simulated synaptic input patterns were quantified by the instantaneous spike rate that was calculated as the inverse of each interspike interval and was averaged across 5-10 repetitions of the stimulus.

\section{RESULTS \\ Plasticity in the STN-GP pathway}

Using in vitro whole-cell recordings, we characterized the STP of excitatory inputs onto rat GP neurons. Postsynaptic currents were measured in voltage clamp at -90 to $-100 \mathrm{mV}$ after local electrical stimulation of excitatory inputs while inhibition was blocked with picrotoxin. Each stimulus activated input fibers leading to responses of 20-200 pA peak amplitude. Failures were never seen, indicating that more than one synapse was activated in all cases. Using stochastic stimulation at a mean frequency of $10 \mathrm{~Hz}$, we found that the average response amplitude varied consistently as a function of the preceding stimulation intervals (Fig. 1). At higher stimulation frequencies, it was evident that this STP consisted of the superimposed influences of facilitation and depression of postsynaptic response amplitudes and that facilitation had a shorter time course than depression (Fig. 1).

\section{Modeling short-term plasticity}

Short-term facilitation and depression were quantified for each neuron $(n=21)$ using a simple dynamic model with four param- 
A
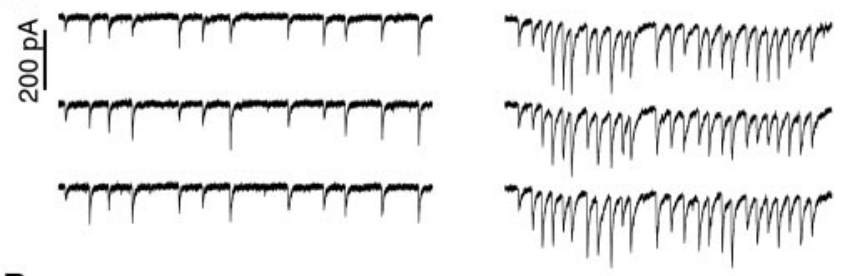

B

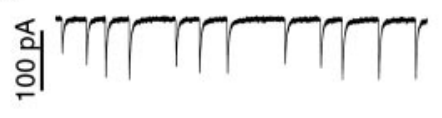

C

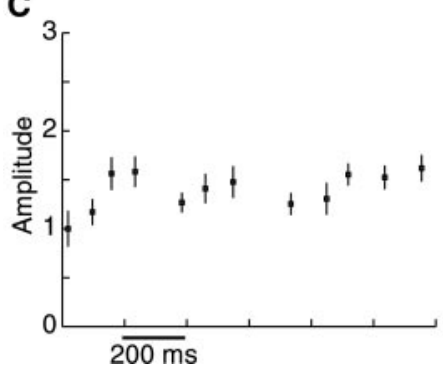

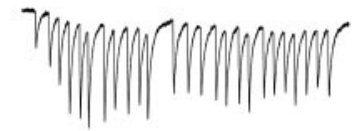

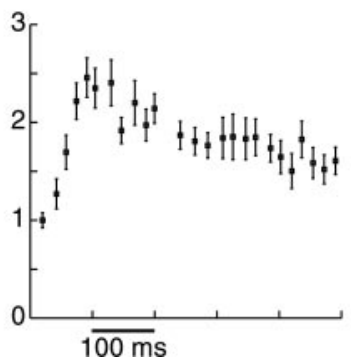

Figure 1. GP neuron responses to excitatory input stimulation. EPSCs were obtained with voltage clamping at a holding potential of $-90 \mathrm{mV}$. Stimulus artifacts were digitally removed. $A$, Individual EPSCs in response to mean $10 \mathrm{~Hz}$ (left) and mean $50 \mathrm{~Hz}$ (right) stimulation. Note that there is considerable variability in the response to particular stimuli in the pattern, but no failures are seen, indicating the stimulation of a small number of afferents. $B$, Averaged EPSCs of 12 repetitions of the same stimulus patterns. $C$, Averaged peak amplitudes (mean \pm SEM) after subtracting the effect of temporal summation. Amplitude measurements of the averages in this and subsequent figures were normalized to the amplitude of the first response after a several second pause in stimulation.

eters: strength of facilitation and depression $\left(\mathrm{Inc}_{\mathrm{F}}\right.$ and $\left.\mathrm{Inc}_{\mathrm{D}}\right)$ and time constant of facilitation and depression $\left(\tau_{\mathrm{F}}\right.$ and $\tau_{\mathrm{D}}$ ) (for details, see Materials and Methods). To examine the influence of STP across a wide range of input rates, we recorded response amplitudes during stochastic stimulation at mean rates of 10, 20, 50 , and $100 \mathrm{~Hz}$ and determined the single best fit of the model across all stimulation frequencies for each neuron. We found that 15 of 21 neurons showed an initial facilitation that was followed by depression. Depending on the relative strength of facilitation and depression, the response to sustained high-frequency stimulation could remain elevated over baseline $(n=7)$ (Figs. 2, 3A, top traces) or show a net suppression $(n=8)$ (Figs. 2, 3A, middle traces). Finally, 7 of 21 neurons showed net depression for all responses (Figs. 2, 3A, bottom traces). Even for these neurons, however, a better fit to the data was produced by the model that included both a facilitating and a depressing variable than by models using only depressing variables (data not shown). This indicates that all observed amplitude profiles resulted from a balance of depressing and facilitating influences (Fig. 2).

The distribution of facilitation and depression parameters for all neurons $(n=21)$ (Fig. $3 B)$ showed that the time course and strength of facilitation and depression were quite variable across neurons. Although STP parameters of the computational model did not reflect underlying physiological mechanisms, the overall balance of facilitation and depression in the model fits the time course of the observed STP quite well. The mean decay time of facilitation was $275 \mathrm{msec}$, whereas depression lasted significantly longer (628 msec; $p<0.001$; Mann-Whitney $U$ test). To examine

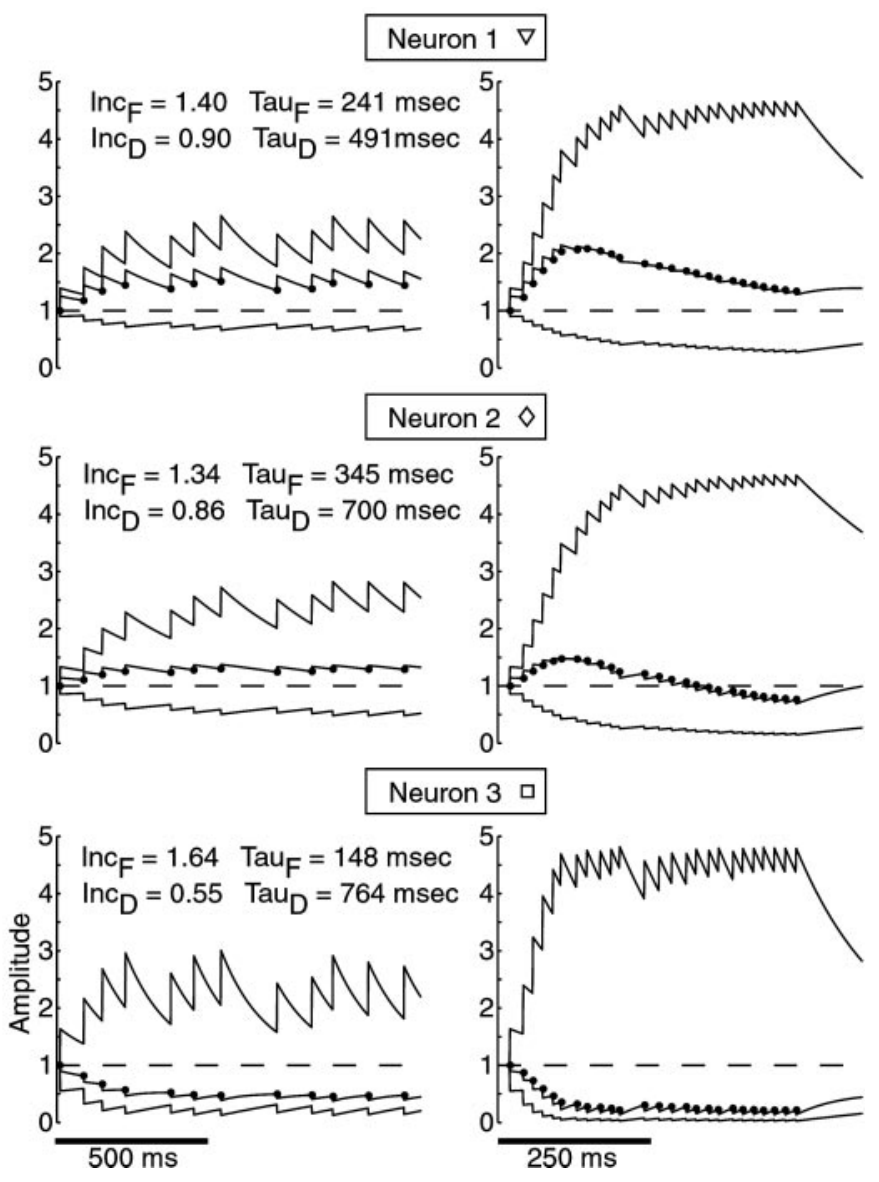

Figure 2. The time course of facilitation and depression parameters describing STP. Three different neurons with distinct profiles of plasticity (square, diamond, and triangle match subsequent figures) are shown in response to mean $10 \mathrm{~Hz}$ (left) and mean $50 \mathrm{~Hz}$ (right) stimulation. In each panel, the top trace depicts the $F$ variable, and the bottom trace depicts the $D$ variable. The multiplication of the two dynamic variables is shown in the middle, and the amplitudes and times of predicted stimulation responses are indicated with filled circles. The values of the model parameters used to generate the traces for each neuron are listed for each neuron. Note that a single parameter fit was used across all stimulation frequencies for each neuron.

whether different physiological types of GP neurons (Kita and Kitai, 1991; Nambu and Llinas, 1994; Cooper and Stanford, 2000) may be associated with specific ranges of STP parameters, we plotted the STP parameters against physiological parameters that have been used to classify types of GP neurons, namely, input resistance $(295 \pm 109 \mathrm{M} \Omega$ ), waveform of spike afterhyperpolarization (monophasic and biphasic), and the amplitude of voltage sags with hyperpolarizing pulses indicative of h current (steadystate voltage response ranged from 57 to $98 \%$ of peak response to $-0.25 \mathrm{nA}$ current injection). Although the full range of values reported previously in these parameters was found in our sample, there was no correlation of these values with STP parameters from the dynamic variable model fits. In addition, we grouped physiological measurements of STP according to the three types of amplitude profiles discussed above and also found no correlation with the postsynaptic membrane properties of GP neurons.

To examine the possibility that the observed STP profiles could be partly mediated by the activation of modulatory fibers such as dopamine or the activation of metabotropic receptors at GABA and glutamate synapses, we performed an additional set of ex- 
A
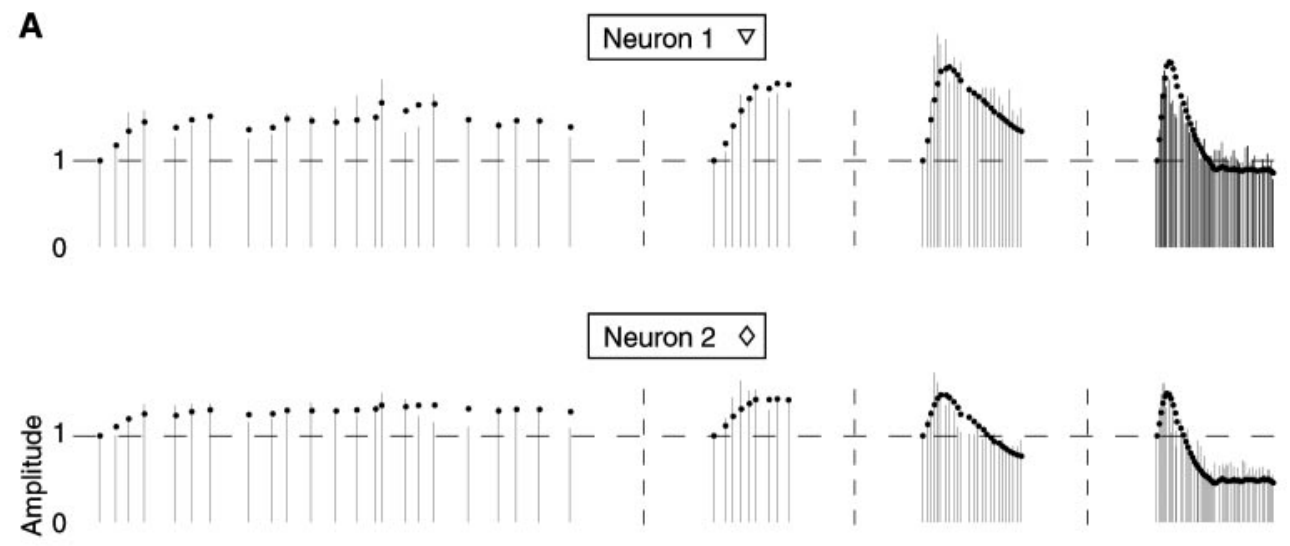

Figure 3. Response amplitudes and model fits across all input frequencies used. $A$,Vertical bars represent response amplitudes at the time of each stimulus. Gray dots indicate the amplitudes produced by the dynamic variable model. The same three neurons as in Figure 2 are shown and identified with the same symbols (square, diamond, and triangle). Neuron 3 व

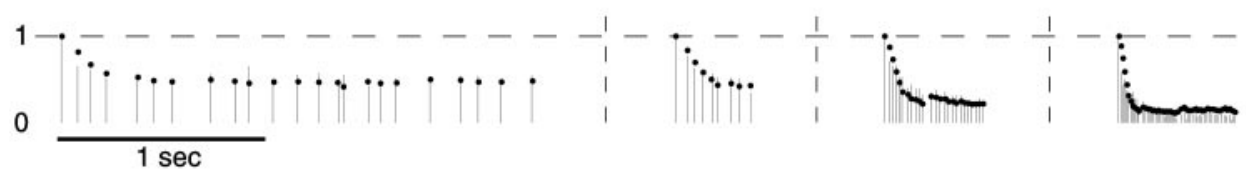
Horizontal dashed lines indicate the level of response amplitude after several seconds of pausing stimulation, and vertical dashed lines indicate breaks between the stimulation sequences. Representative portions of the stimulation protocol to which the model was fit are shown (left to right) for stochastic $10,20,50$, and 100 $\mathrm{Hz}$ stimulation. $B$, Distribution of model parameters from fits of inputs to 21 different neurons. Inc $\mathrm{F}_{\mathrm{F}}$ and $\tau_{\mathrm{F}}$ values from each neuron are plotted against each other (left), and $\operatorname{Inc}_{\mathrm{D}}$ and $\tau_{\mathrm{D}}$ values from each neuron are plotted against each other (right). Note the continuous distribution of each of the four parameters. Data points corresponding to the three example neurons used throughout the article are indicated with squares, diamonds, and triangles.

periments, in which STP was measured before and after washing in a mixture of receptor antagonists. Specifically, dopamine receptors were blocked with $10 \mu \mathrm{M}$ haloperidol (Research Biochemicals, Natick, MA), GABA ${ }_{\mathrm{B}}$ receptors were blocked with 0.5 $\mu \mathrm{M}$ CGP52432 (Tocris Cookson, Ballwin, MO), group I metabotropic glutamate receptors (mGluRs) were blocked with a combination of $50 \mu \mathrm{M}$ LY367385 and $0.5 \mu \mathrm{M}$ MPEP hydrochloride, and group II/III mGluRs were blocked with $0.5 \mu \mathrm{M}$ CPPG (all obtained from Tocris Cookson). The overall profiles of STP did not differ significantly before and after the application of these drugs ( $n=5$ neurons), as illustrated by the responses of each neuron to the $50 \mathrm{~Hz}$ burst portion of the stimulus protocol (Fig. 4). Dynamic variable model fits of STP parameters to the responses of each neuron after application of the antagonist mixture fell within the range of parameters observed in the original set of recordings shown in Figure $3 B$. To quantify possible small differences in STP profiles before and after antagonist application, we fit the dynamic variable model to response amplitudes after drug application and examined the quality of the model in describing responses before drug application. The root mean square error of the model fit increased only slightly, from $15.5 \pm 1.9 \%$ (mean \pm SEM) to $19.7 \pm 3.3 \%$, when the model was used to fit predrug amplitudes. This good cross-fit supports the conclusion that the antagonists caused no change in STP, indicating that the possible stimulation of modulatory synapses did not have a noticeable influence on our measurements of STP.

Additional control experiments were performed to determine whether voltage escape at the site of synaptic input and the activation of voltage-gated conductances could be contributing to the observed properties of synaptic plasticity. In these experiments, postsynaptic voltage-gated sodium and potassium currents were blocked by including $10 \mathrm{~mm}$ QX-314 and replacing potassium with cesium in the recording pipette. The short-term plasticity profiles under these conditions retained both facilitation and depression parameters in the same range as observed in the absence of channel blockers (Fig. 5). For two of six neurons, recorded depression was dominant, whereas the other four neurons showed net facilitation at the beginning and depression toward the end of $50 \mathrm{~Hz}$ stimulus sequences. Responses to the 50 $\mathrm{Hz}$ portion of the stimulus protocol at holding potentials of -70 , -90 , and $-110 \mathrm{mV}$ showed no nonlinearities, indicative of voltage escape or the activation of voltage-gated channels. These results indicate that neither facilitation nor depression in our recordings was caused to a significant degree by postsynaptic voltage escape. For holding potentials more depolarized than $-70 \mathrm{mV}$, signs of such escape were indeed present, however, indicating that active properties under normal levels of depolarization are likely to play an important role in synaptic integration in these cells.

\section{Short-term plasticity and rate coding}

Previous studies have shown that STP can have a dramatic effect on rate coding, i.e., on the translation of presynaptic activity rates into the postsynaptic output spike rate. In cases of strong short- 
A
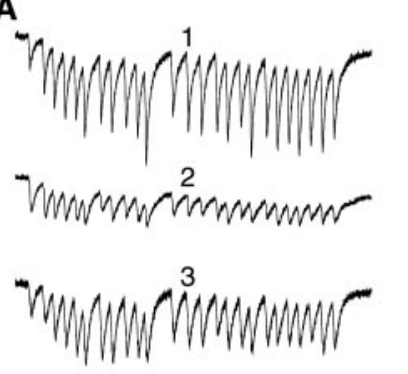

7numurumunumur

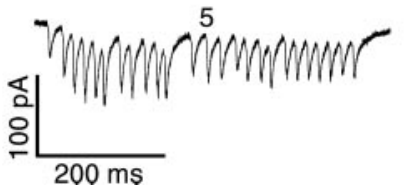

B
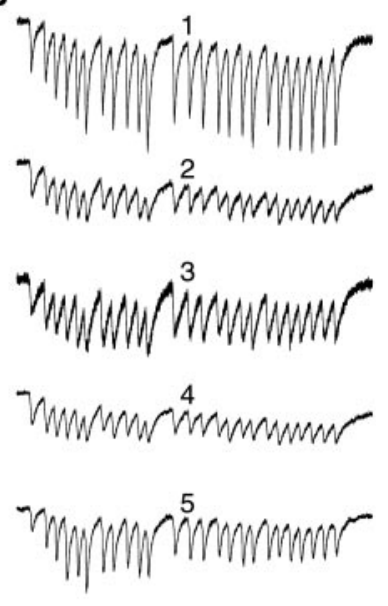

Figure 4. Responses of five GP neurons to $50 \mathrm{~Hz}$ stimulation before and after application of dopamine receptor, $\mathrm{GABA}_{\mathrm{B}}$ receptor, and $\mathrm{mGluR}$ antagonists (see Results). $A$, EPSCs recorded before drug wash-in. $B$, EPSCs recorded after application of the antagonists. To allow for a better visual comparison between neurons, the traces of neurons 2-5 were normalized by a constant scale factor for each neuron such that the amplitude of the first EPSC in $A$ matched the response of neuron 1. Thus, the amplitude scale bar needs to be multiplied by the scale factors of 1.19 , $1.45,0.98$, and 1.54 for neurons $2-5$, respectively. Note the similar amplitude profiles of each neuron before and after drug application.

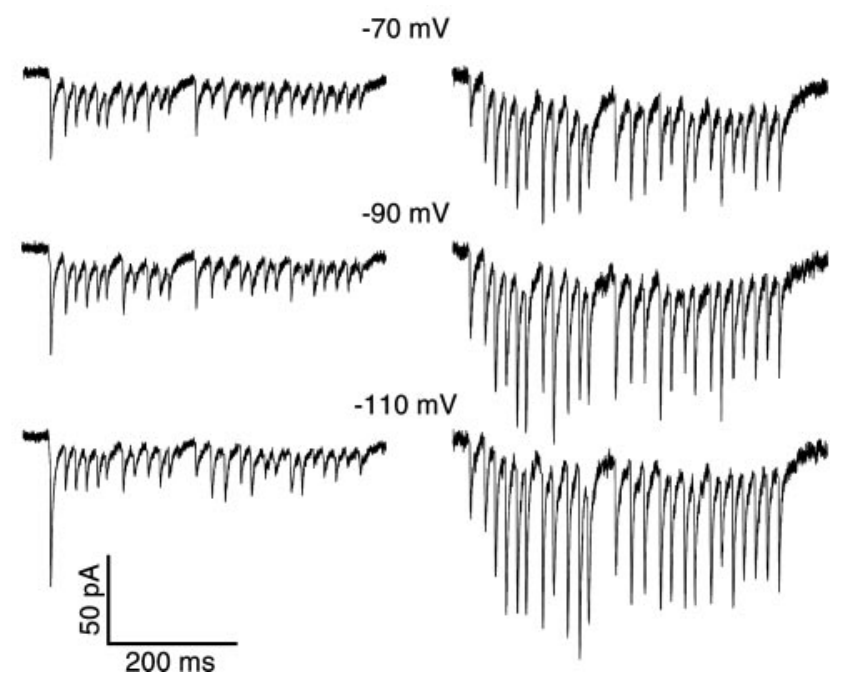

Figure 5. Responses of two GP neurons (left and right columns) to $50 \mathrm{~Hz}$ stimulation in the presence of QX-314 and cesium in the intracellular recording solution. Responses are shown at $-70,-90$, and $-110 \mathrm{mV}$ holding potentials.

term depression of excitatory inputs, the postsynaptic spike rate might become independent of the presynaptic input rate altogether (Abbott et al., 1997), challenging the concept of a linear relationship between input and output rates underlying the present circuit model of the basal ganglia. Because the in vitro stimulation techniques used to characterize synaptic plasticity can activate only a few of the hundreds of STN inputs to each GP neuron, electrical stimulation could not be used to activate populations of synaptic input representative of the complete excitatory drive to a GP neuron in vivo. Therefore, we used the

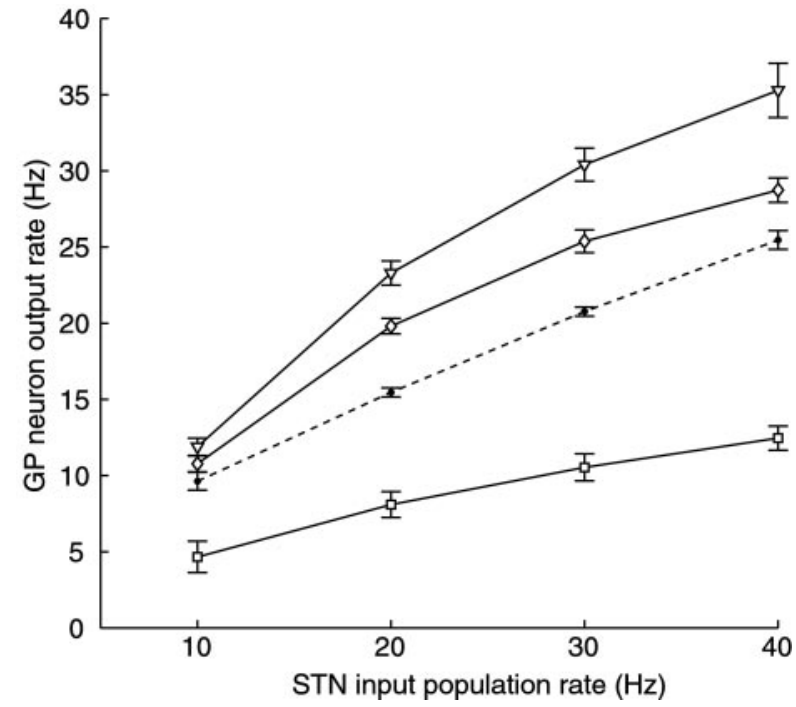

Figure 6. The rate-coding function of GP neurons $(n=6)$ for excitatory input under the influence of three example plasticity profiles. Steady-state GP neuron response rates (mean \pm SEM) during dynamic current clamping are plotted as a function of the rate of the simulated STN input population. For details, see Results. The case of no plasticity is indicated with a dashed line, and the example STP profiles (from the fits of Fig. 3) are identified with squares, diamonds, and triangles.

technique of dynamic clamping, in which arbitrary synaptic conductance patterns can be applied to neurons in vitro (see Materials and Methods), to determine how the effect of specific input patterns may be modulated by STP.

To determine the influence of presynaptic activity rate on output spiking in the presence of STP, we simulated 100 separate STN inputs all firing independently with a random pattern at mean rates of $10,20,30$, or $40 \mathrm{~Hz}$, representing the range of sustained steady-state firing rates seen in vivo in the rat (Magill et al., 2000; Urbain et al., 2000). To examine the effect of STP, we created three different postsynaptic conductances that were derived from identical input times but were modulated by applying the STP parameters from our three prototypical example neurons shown in Figures 2 and $3 A$. For comparison, an excitatory conductance without STP was also generated. To measure the influence of STP on rate coding, average GP firing rates were measured at each level of STN input firing after steady-state firing was reached (Fig. 6). Although there was still an increase in spike rate with increasing input rate for all forms of STP, the increase in spike rate was much reduced for the case of strong depression. Even at a slow rate of $10 \mathrm{~Hz}$ input activity, synapses dominated by depression slowed the output spiking to $<50 \%$ of the default value without STP. The cases of mixed net facilitation and depression led to a nonlinear rate-coding function, which showed a steeper slope at low input rates than at high input rates (Table 1). These findings indicate that STP significantly modulates the ratecoding function of excitatory input to GP and that increases in input rate may affect some GP neurons much differently from others based on the STP parameters in the inputs.

\section{Short-term plasticity and temporal coding}

Strong phasic temporal modulations in STN activity are present during movement (Georgopoulos et al., 1983; DeLong et al., 1985; Wichmann et al., 1994). In the parkinsonian state, STN activity becomes bursty in rats (Hollerman and Grace, 1992; Hassani et al., 1996; Hassani and Feger, 1999; Ni et al., 2001), 


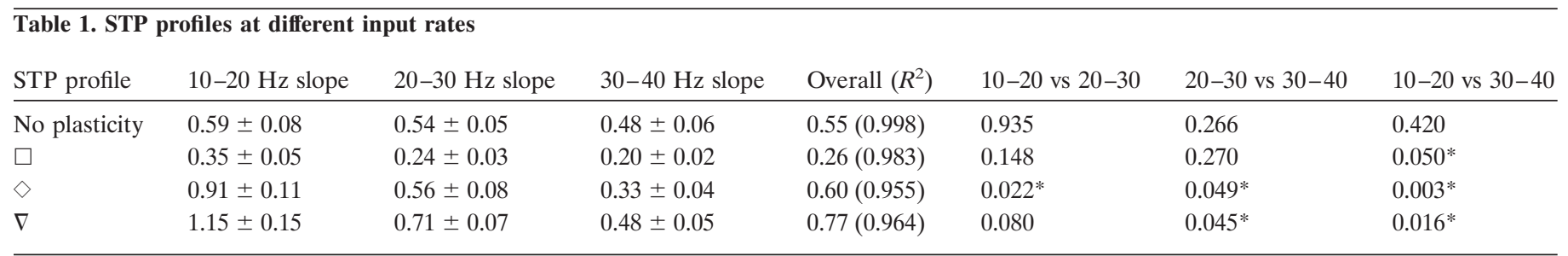

The square, diamond, and triangle are explained in Figure 2.

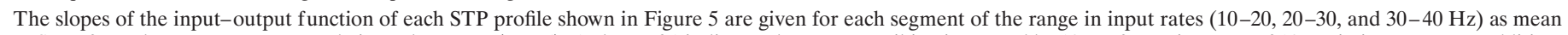

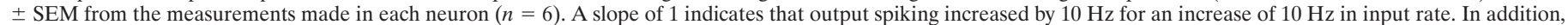

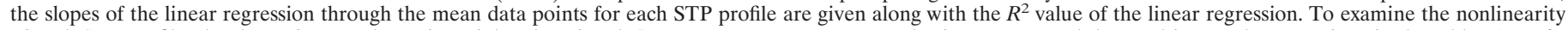

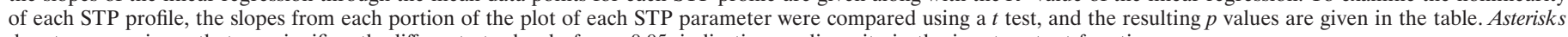
denote comparisons that are significantly different at a level of $\alpha=0.05$, indicating nonlinearity in the input-output function.

A
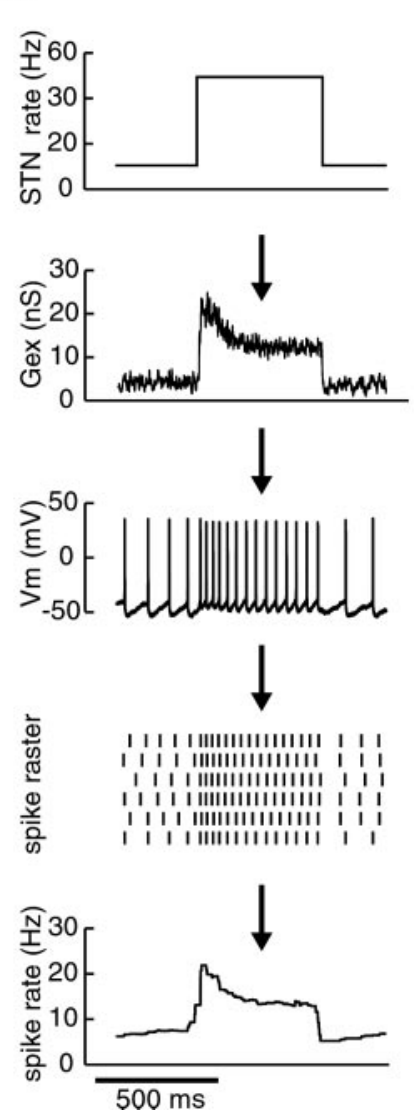

B
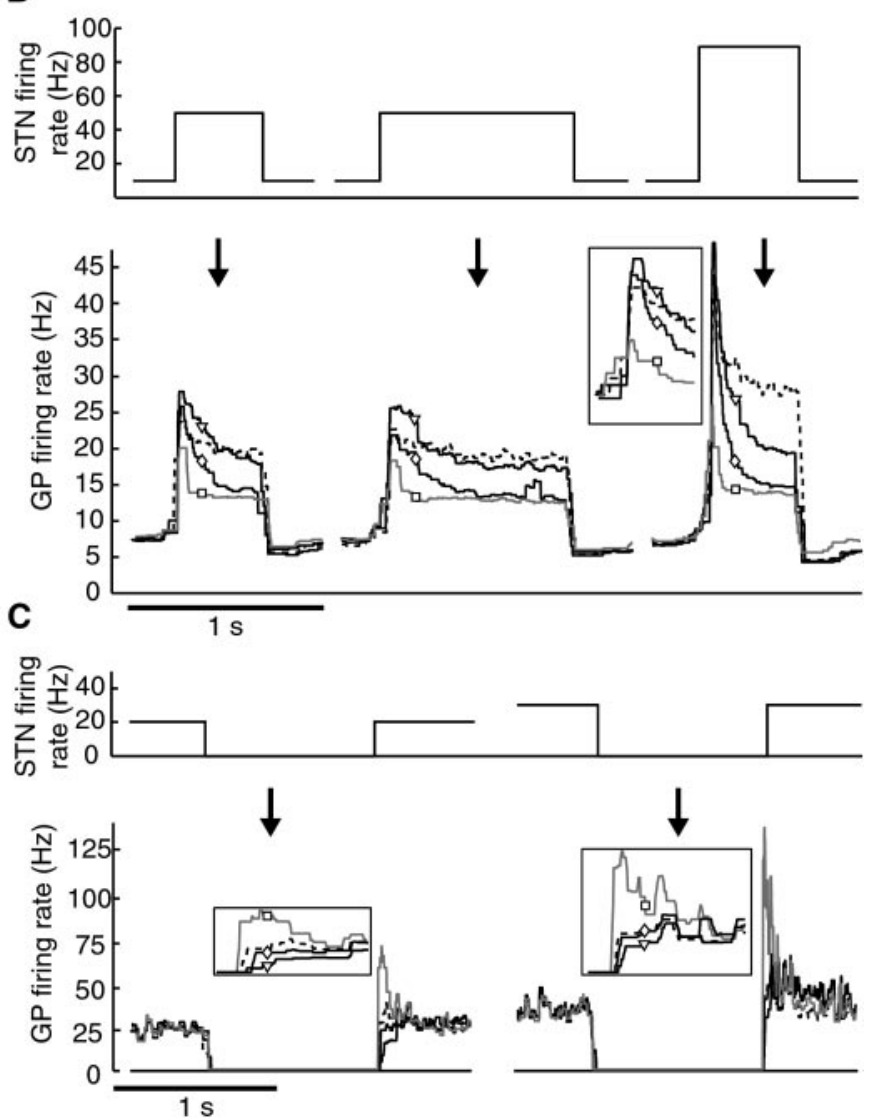

Figure 7. The temporal-coding function of STP for simulated STN input patterns to GP neurons. Input patterns were constructed as described in Results. To allow a comparison of the influence of the different types of plasticity on spike rate during phasic bursts and pauses in input activity, all of the excitatory conductances were normalized so that the mean levels preceding a burst or a pause were the same for each profile of plasticity. $A$, Illustration of how the input-output function was characterized. Top to bottom, Mean STN input population rates were used to generate total excitatory conductance traces with a given plasticity profile applied $\left(G_{\mathrm{ex}}\right)$. GP neuron membrane potential $\left(V_{\mathrm{m}}\right)$ was measured during application of the stimulus using dynamic current clamping (see Materials and Methods). Spike times were determined across repeated applications of the same input conductance (spike raster), and the resulting instantaneous GP neuron output spike rate was determined. $B$, Typical input-output function of a GP neuron in response to bursty STN input activity. GP spike rates during the influence of the prototypical STP profiles are indicated with squares, diamonds, and triangles and are shown in light gray, medium gray, and black, respectively. The case of no plasticity is indicated with a dashed line. The enhancement of burst onsets was greatest at higher burst frequencies, and steady-state response level was reached $\sim 500 \mathrm{msec}$ into the bursts in all neurons tested $(n=8)$. The inset shows the initial portion of the responses to the $100 \mathrm{~Hz}$ burst at a $0.5 \times$ rate scale and $4 \times$ time scale. $C$, Input-output function during pauses in STN input activity. The different plasticity profiles are represented as in $B$. Responses immediately after the pauses are shown in the inset at a $0.5 \times$ rate scale and $4 \times$ time scale. The response to pause offsets seen with the STP profile dominated by depression (square) was greatest at higher baseline frequencies, and the highest response level was reached after the end of 1 sec pauses in all neurons tested ( $n=7)$. Arrows indicate stepwise transformation from input rate to output rate.

monkeys (Bergman et al., 1994), and humans (Levy et al., 2000). The synaptic decoding of these STN activity patterns in GP can be expected to be modulated by STP. We examined this hypothesis by constructing presynaptic activity patterns that contained bursts of STN activity based on data from recordings in rats (Hassani et al., 1996; Hassani and Feger, 1999; Ni et al., 2001). We generated generic examples of bursts and pauses with durations and spike rates representing the range of in vivo recordings to determine the effect of STP on temporal coding for a set of well defined input parameters. Based on in vivo recordings from anesthetized rats (Magill et al., 2000), we assumed that bursts in different STN cells are highly correlated with each other. Again, simulated inputs were made incorporating each of the three example STP profiles shown in the previous experiments in addition to a control condition with no plasticity.

The pattern of GP neuron responses to different STP conditions was quantified by examining the instantaneous GP neuron output firing rate across repeated applications of each input condition (Fig. 7A). The results indicate that STP significantly modulates the GP response to synchronous bursts and pauses in STN firing. The response to the onset of bursts was enhanced by facilitation, whereas the continued response during an input burst was highly dampened by depression (Fig. 7B). Examination of the different STP profiles showed that all combinations of facilitation 


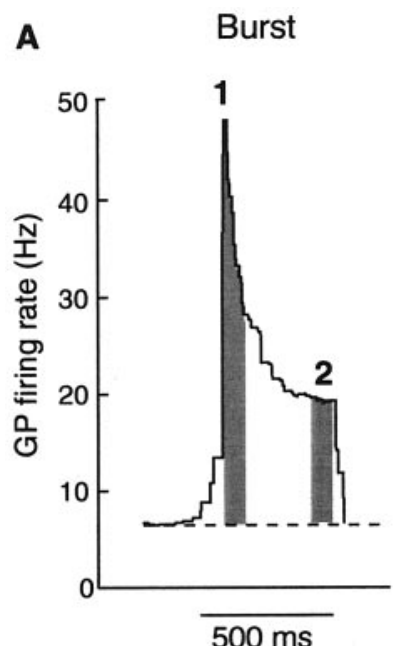

C Pause
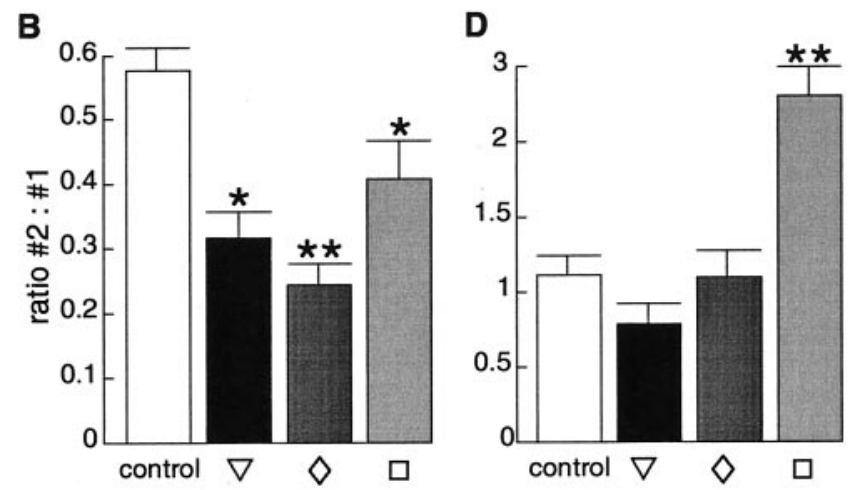

Figure 8. Temporal dynamics of GP spike rates during bursts and pauses in STN input with different plasticity profiles. Spike rates were calculated during dynamic current clamping of simulated STN input as described in Figure 5. $A$, Increases in GP spike rate were measured during the first 100 msec and last $100 \mathrm{msec}$ of $500 \mathrm{msec}$ bursts in STN input. The example shown is from the STP profile denoted by a diamond, and time windows in which spiking was measured are indicated in gray and labeled as 1 and 2. $B$, The ratio of these two portions of the burst response were determined for each plasticity profile in each neuron $(n=8$; data shown as mean \pm SEM). This ratio for each of the prototypical STP profiles was significantly different from the control condition of no plasticity $\left({ }^{*} p<\right.$ $0.05 ;{ }^{*} p<0.01$; Mann-Whitney $U$ test). $C$, Spike rates measured during the $100 \mathrm{msec}$ preceding and $100 \mathrm{msec}$ immediately after a pause in STN input. The example shown is from the STP profile denoted by a square, and areas from which spiking was measured are indicated as in $A$. $D$, The ratio of these two portions of the spike pattern were determined in the same manner as in $B$. Only one of the profiles of STP had a ratio significantly different ratio from the control condition of no plasticity.

and depression resulted in significant increases of the relative decrease in GP spike rate between the start and end of input bursts compared with the case of no plasticity (Fig. $8 \mathrm{~B}$ ). Even in the absence of STP, however, responses to burst inputs were enhanced at the onset and adapted thereafter (Fig. 7B). This indicates that intrinsic active properties and STP properties support each other in this regard.

The GP response after pauses of STN input was strongly affected only by the STP profile dominated by depression (Fig. $8 C, D)$, which led to enhanced responses after a pause in STN firing attributable to the removal of depression during the pause interval (Fig. 7C). Overall, these results suggest that a main role of STP in the STN-GP pathway may be to enhance the edges of responses to increases in STN activity while disallowing prolonged burst responses to input bursts.

\section{DISCUSSION}

We found both short-term depression and facilitation in excitatory inputs to GP neurons. Different GP neurons showed a range of STP parameters, but depression generally lasted longer than facilitation and therefore dominated in most cases. Although previous studies have proposed the existence of different subtypes of GP neurons based on heterogeneous membrane properties (Kita and Kitai, 1991; Nambu and Llinas, 1994; Cooper and Stanford, 2000), there was no relationship of the STP profiles we observed to the intrinsic physiological properties of the recorded neurons. This indicates that the heterogeneity observed in STP is not related to physiological subpopulations of GP neurons but is most likely a consequence of properties in the STN synaptic terminals in GP. Because we found these STP properties consistently in all recordings, we are confident that they are properties of the massive projection from STN (Shink and Smith, 1995; Shink et al., 1996; Smith et al., 1998), although we cannot exclude the possibility that we might have stimulated projections from cortex and thalamus to GP in a few cases. The activation of multiple input sources in all of our recordings is highly unlikely, because the stimulation electrodes used activated only a few fibers in a very small region (see Materials and Methods).

A potential concern is that stimulation of axons severed from their origin in STN results in synaptic depression caused by presynaptic degradation. This seems unlikely, however, because previous studies using high-frequency stimulus-train protocols have demonstrated the presence of both short-term depression and facilitation in preparations in which the cell bodies of synaptic sources are either present or absent from the slice (Varela et al., 1997; Dittman et al., 2000; Hempel et al., 2000). In addition, the consistent size of EPSCs and profile of STP during many repeated stimulations of each input sequence over $>30$ min duration in our experiments indicate that STN terminals did not show any signs of irreversible rundown.

The considerable range of STP parameters found suggests that STP between STN and GP itself may undergo plastic changes. Such metaplasticity of STP profiles has been reported in cortex (Finnerty et al., 1999) and hippocampus (Goussakov et al., 2000) and may have important functional implications (Nadim and Manor, 2000). It is interesting to speculate that particular STP profiles may be learned in specific populations of functionally connected neurons in the STN-GP pathway to control temporal pattern generation during behavior. This function would be well suited to aid in the proposed role of the STN-GP interaction in pattern generation and the control of movement sequences (Berns and Sejnowski, 1998; Plenz and Kitai, 1999; Magill et al., 2000). The mechanisms by which STP properties could be adapted over time probably would involve neuromodulators (Gil et al., 1997; Parker and Grillner, 1999) or be caused by changes in the state of long-term potentiation or depression of a given synapse (Markram and Tsodyks, 1996). Because disease states such as Parkinson's disease involve alterations in neuromodulation and probably long-term plasticity, it is possible that STP properties also become changed during disease states. It would therefore be interesting to examine STP in such conditions to determine its possible involvement in the alteration of activity patterns.

The classic model of basal ganglia function and dysfunction assumes a simple relationship between input and output rates of basal ganglia structures (Albin et al., 1989; DeLong, 1990). We show that, because of the presence of STP, the relationship 
between input and output rates may be quite heterogeneous among GP neurons. Synaptic connections that show prominent facilitation amplify presynaptic rate changes, whereas prominent depression nearly suppresses any change in postsynaptic rates. A uniform effect of rate changes in a basal ganglion structure on connected structures should therefore not be expected. The presence and properties of STP in other basal ganglia interconnections remains to be determined and could bring further into question the classic model of basal ganglion dysfunction. In the present study, we focused on STP of the excitatory input to GP, because bursting patterns in the STN input to GP have been measured in normal and disease states and are likely to be highly significant in the control of GP spiking. The effectiveness of deep brain stimulation in the STN in changing activity patterns in Parkinson's disease highlights the importance of this connection. In vivo, however, the excitatory drive of STN on GP will be counteracted by inhibition from the striatum, as well as collaterals of GP neurons (Shink and Smith, 1995; Shink et al., 1996; Smith et al., 1998). These inhibitory connections may also show STP, which could further modify the response to temporal input patterns.

In addition to the influence of STP on rate coding, we showed that temporal activity patterns in the STN-GP pathway are significantly modulated by STP. These effects consisted of an increase in the response to onsets of input bursts, whereas the response to maintained input bursts was dampened. Furthermore, STP profiles with dominant depression were associated with a rebound response after a pause in STN input. These effects provide a potential mechanism to filter temporal response profiles within the basal ganglia loop. Intrinsic properties of GP neurons were also clearly responsible for nonlinear components to stimulus responses in our dynamic clamp experiments. Thus, it is an interaction of intrinsic active properties and synaptic input properties that ultimately determines the emerging activity patterns under any given condition. Although these mechanisms can be individually identified and measured in biological preparations, we believe that biophysically accurate computer modeling will be necessary to make quantitative statements about the effect of complex dynamic interactions between all variables.

Dynamic clamping allows the application of arbitrary conductance patterns to neurons and thus also aids in determining complex interactions between intrinsic properties and synaptic input. This technique is limited, however, in that all conductances are applied at a single point. Nevertheless, active dendritic properties can still be evoked with a somatic clamp because of the considerable spread of current from the soma into the dendrites. In a simulation study of cerebellar Purkinje cells, we showed that large dendritic currents were activated in the same manner by distributed dendritic synaptic inputs as by the same conductances focalized at the soma (Jaeger et al., 1997). In recent modeling work, we showed that, in GP neurons, purely somatic input also interacts with dendritic currents to a large degree (Jaeger, 2001). Nevertheless, specific interactions with dendritic inputs that lead to large local voltage transients may be lost with dynamic clamping. For example, voltage-gated sodium channels that we identified in GP dendrites (Hanson et al., 2001) could lead to an all-or-none amplification of distal AMPA inputs. STP in this condition could be crucial in leading to suprathreshold responses for specific portions of input sequences. As the details of voltagegated ion channel contribution to dendritic processing of synaptic inputs in GP are worked out using anatomic and physiological methods, we will use computer-simulated multicompartmental models of GP neurons to further elucidate the interaction of STP with active dendritic membrane properties.

The STN-GP feedback connection has been the focus of interest in understanding pattern generation in the basal ganglia (Plenz and Kitai, 1999), may be involved in forming sequence memories (Berns and Sejnowski, 1998), and, in Parkinson's disease, is implicated in the generation of tremor (Hurtado et al., 1999; Levy et al., 2000). The generation of oscillations in the STN-GP network probably depends on the details of the anatomic connectivity, as well as intrinsic conductances. The effects of short-term plasticity described in the present study further modify oscillations and feedback patterns in these structures. For example, the dampening of the response to prolonged bursts of STN input caused by depression may account for the findings that GP neurons are often less bursty than their STN counterparts (Magill et al., 2000; Urbain et al., 2000). Because oscillations in the STN-GP system are known to correlate with tremor in Parkinson's disease (Hurtado et al., 1999; Levy et al., 2000), prevention of these oscillations could provide a treatment for tremor. As the details of the mechanisms underlying STP are elucidated, it may be possible to develop drugs that alter the expression of short-term depression and facilitation in the STN-GP connection, leading to a suppression of tremor.

\section{REFERENCES}

Abbott LF, Varela JA, Sen K, Nelson SB (1997) Synaptic depression and cortical gain control. Science 275:220-224.

Albin RL, Young AB, Penney JB (1989) The functional anatomy of basal ganglia disorders. Trends Neurosci 12:366-375.

Bergman H, Wichmann T, Karmon B, DeLong MR (1994) The primate subthalamic nucleus. II. Neuronal activity in the MPTP model of parkinsonism. J Neurophysiol 72:507-520.

Bergman H, Feingold A, Nini A, Raz A, Slovin H, Abeles M, Vaadia E (1998) Physiological aspects of information processing in the basal ganglia of normal and parkinsonian primates. Trends Neurosci 21:32-38.

Berns GS, Sejnowski TJ (1998) A computational model of how the basal ganglia produce sequences. J Cognit Neurosci 10:108-121.

Beurrier C, Congar P, Bioulac B, Hammond C (1999) Subthalamic nucleus neurons switch from single-spike activity to burst-firing mode. J Neurosci 19:599-609.

Bonci A, Malenka RC (1999) Properties and plasticity of excitatory synapses on dopaminergic and GABAergic cells in the ventral tegmental area. J Neurosci 19:3723-3730.

Cooper AJ, Stanford IM (2000) Electrophysiological and morphological characteristics of three subtypes of rat globus pallidus neurone in vitro. J Physiol 527:291-304.

DeLong MR (1990) Primate models of movement disorders of basal ganglia origin. Trends Neurosci 13:281-285.

DeLong MR, Crutcher MD, Georgopoulos AP (1985) Primate globus pallidus and subthalamic nucleus: functional organization. J Neurophysiol 53:530-543.

Deschenes M, Bourassa J, Doan VD, Parent A (1996) A single-cell study of the axonal projections arising from the posterior intralaminar thalamic nuclei in the rat. Eur J Neurosci 8:329-343.

Destexhe A, Rudolph M, Fellous JM, Sejnowski TJ (2001) Fluctuating synaptic conductances recreate in vivo-like activity in neocortical neurons. Neuroscience 107:13-24.

Dittman JS, Kreitzer AC, Regehr WG (2000) Interplay between facilitation, depression, and residual calcium at three presynaptic terminals. J Neurosci 20:1374-1385

Filion M, Tremblay L (1991) Abnormal spontaneous activity of globus pallidus neurons in monkeys with MPTP-induced parkinsonism. Brain Res 547:142-151.

Finnerty GT, Roberts LS, Connors BW (1999) Sensory experience modifies the short-term dynamics of neocortical synapses. Nature 400:367-371.

Gardiner TW, Kitai ST (1992) Single-unit activity in the globus pallidus and neostriatum of the rat during performance of a trained head movement. Exp Brain Res 88:517-530.

Gauck V, Jaeger D (2000) The control of rate and timing of spikes in the deep cerebellar nuclei by inhibition. J Neurosci 20:3006-3016.

Georgopoulos AP, DeLong MR, Crutcher MD (1983) Relations between parameters of step-tracking movements and single cell discharge in the globus pallidus and subthalamic nucleus of the behaving monkey. J Neurosci 3:1586-1598. 
Gil Z, Connors BW, Amitai Y (1997) Differential regulation of neocortical synapses by neuromodulators and activity. Neuron 19:679-686.

Goussakov IV, Fink K, Elger CE, Beck H (2000) Metaplasticity of mossy fiber synaptic transmission involves altered release probability. J Neurosci 20:3434-3441.

Hanson J, Jaeger D, Smith Y (2001) Subcellular localization of sodium channels in the rat globus pallidus and dendritic boosting of synaptic inputs. Soc Neurosci Abstr 27:257.

Hassani OK, Feger J (1999) Effects of intrasubthalamic injection of dopamine receptor agonists on subthalamic neurons in normal and 6-hydroxydopamine-lesioned rats: an electrophysiological and c-Fos study. Neuroscience 92:533-543.

Hassani OK, Mouroux M, Feger J (1996) Increased subthalamic neuronal activity after nigral dopaminergic lesion independent of disinhibition via the globus pallidus. Neuroscience 72:105-115.

Hempel CM, Hartman KH, Wang XJ, Turrigiano GG, Nelson SB (2000) Multiple forms of short-term plasticity at excitatory synapses in rat medial prefrontal cortex. J Neurophysiol 83:3031-3041.

Hollerman JR, Grace AA (1992) Subthalamic nucleus cell firing in the 6-OHDA-treated rat: basal activity and response to haloperidol. Brain Res 590:291-299.

Houck CR, Joines JA, Kay MG (1995) A genetic algorithm for function optimization: a Matlab implementation. North Carolina State University-Industrial Engineering Department Tech Report 95:1-14. www.ie.ncsu.edu/gaot/GAToolBox/gaot.

Hurtado JM, Gray CM, Tamas LB, Sigvardt KA (1999) Dynamics of tremor-related oscillations in the human globus pallidus: a single case study. Proc Natl Acad Sci USA 96:1674-1679.

Jaeger D (2001) Synaptic shunting of membrane currents in different neural morphologies. Soc Neurosci Abstr 27:256.

Jaeger D, Bower JM (1999) Synaptic control of spiking in cerebellar Purkinje cells: dynamic current clamp based on model conductances. J Neurosci 19:6090-6101.

Jaeger D, Gilman S, Aldridge JW (1995) Neuronal activity in the striatum and pallidum of primates related to the execution of externally cued reaching movements. Brain Res 694:111-127.

Jaeger D, De Schutter E, Bower JM (1997) The role of synaptic and voltage-gated currents in the control of Purkinje cell spiking: a modeling study. J Neurosci 17:91-106.

Kita H, Kitai ST (1991) Intracellular study of rat globus pallidus neurons: membrane properties and responses to neostriatal, subthalamic and nigral stimulation. Brain Res 564:296-305.

Levy R, Hutchison WD, Lozano AM, Dostrovsky JO (2000) Highfrequency synchronization of neuronal activity in the subthalamic nucleus of parkinsonian patients with limb tremor. J Neurosci 20:7766-7775.

Magill PJ, Bolam JP, Bevan MD (2000) Relationship of activity in the subthalamic nucleus-globus pallidus network to cortical electroencephalogram. J Neurosci 20:820-833.

Markram H, Tsodyks M (1996) Redistribution of synaptic efficacy between neocortical pyramidal neurons. Nature 382:807-810.

Nadim F, Manor Y (2000) The role of short-term synaptic dynamics in motor control. Curr Opin Neurobiol 10:683-690.
Naito A, Kita H (1994) The cortico-pallidal projection in the rat: an anterograde tracing study with biotinylated dextran amine. Brain Res 653:251-257.

Nambu A, Llinas R (1994) Electrophysiology of globus pallidus neurons in vitro. J Neurophysiol 72:1127-1139.

Ni Z, Bouali-Benazzouz R, Gao D, Benabid AL, Benazzouz A (2001) Intrasubthalamic injection of 6-hydroxydopamine induces changes in the firing rate and pattern of subthalamic nucleus neurons in the rat. Synapse 40:145-153.

Nini A, Feingold A, Slovin H, Bergman H (1995) Neurons in the globus pallidus do not show correlated activity in the normal monkey, but phase-locked oscillations appear in the MPTP model of parkinsonism. J Neurophysiol 74:1800-1805.

Parker D, Grillner S (1999) Activity-dependent metaplasticity of inhibitory and excitatory synaptic transmission in the lamprey spinal cord locomotor network. J Neurosci 19:1647-1656.

Plenz D, Kitai ST (1999) A basal ganglia pacemaker formed by the subthalamic nucleus and external globus pallidus. Nature 400:677-682.

Robinson HP, Kawai N (1993) Injection of digitally synthesized synaptic conductance transients to measure the integrative properties of neurons. J Neurosci Methods 49:157-165.

Sharp AA, O’Neil MB, Abbott LF, Marder E (1993) Dynamic clamp: computer-generated conductances in real neurons. J Neurophysiol 69:992-995.

Shink E, Smith Y (1995) Differential synaptic innervation of neurons in the internal and external segments of the globus pallidus by the GABAand glutamate-containing terminals in the squirrel monkey. J Comp Neurol 358:119-141.

Shink E, Bevan MD, Bolam JP, Smith Y (1996) The subthalamic nucleus and the external pallidum: two tightly interconnected structures that control the output of the basal ganglia in the monkey. Neuroscience 73:335-357.

Smith Y, Bevan MD, Shink E, Bolam JP (1998) Microcircuitry of the direct and indirect pathways of the basal ganglia. Neuroscience 86:353-387.

Turner RS, Anderson ME (1997) Pallidal discharge related to the kinematics of reaching movements in two dimensions. J Neurophysiol 77:1051-1074.

Urbain N, Gervasoni D, Souliere F, Lobo L, Rentero N, Windels F, Astier B, Savasta M, Fort P, Renaud B, Luppi PH, Chouvet G (2000) Unrelated course of subthalamic nucleus and globus pallidus neuronal activities across vigilance states in the rat. Eur J Neurosci 12:3361-3374.

Varela JA, Sen K, Gibson J, Fost J, Abbott LF, Nelson SB (1997) A quantitative description of short-term plasticity at excitatory synapses in layer $2 / 3$ of rat primary visual cortex. J Neurosci 17:7926-7940.

Wichmann T, DeLong MR (1996) Functional and pathophysiological models of the basal ganglia. Curr Opin Neurobiol 6:751-758

Wichmann T, DeLong MR (1998) Models of basal ganglia function and pathophysiology of movement disorders. Neurosurg Clin $\mathrm{N}$ Am 9:223-236.

Wichmann T, Bergman H, DeLong MR (1994) The primate subthalamic nucleus. I. Functional properties in intact animals. J Neurophysiol 72:494-506. 